\title{
15
}

\section{Forging die modelling using rule based system}

\author{
Y. Murakami
}

Sumitomo Metal Industries, Ltd.

4-7-28, Kitahama, Chuo-ku, Osaka, Osaka, 541 Japan

E. Arai

Osaka Univ.

2-1, Yamada-oka, Suita, Osaka, 565 Japan

F. Kimura

Univ. of Tokyo

7-3-1, Hongo Bunkyo, Tokyo, 113 Japan

\begin{abstract}
Three-dimensional CAD/CAM systems are generally used in manufacturing forging molds and dies for automotive crankshafts. Although the conventional process dependent on wooden models has been gradually replaced to some extent by an NC process with still expensive cost, another advanced process then become required for 3D modeling and verification of $\mathrm{NC}$ data since it still greatly depends on the skilled operator's experience.

In this study, examples of an automated CAD system for three-dimensional surface modeling with a rule-based modeling process are introduced. This uses intelligent $\mathrm{CAD}$ construction tools in crankshaft forgings and dies.
\end{abstract}

Keywords

Intelligent CAD, crank-shaft, modeling process, rule-based, object-oriented programming 
Three-dimensional CAD/CAM systems are used in mechanical parts industries to define $3 \mathrm{D}$ geometry of automotive crankshafts. However, due to the increasing demand from automotive companies for lighter-weight crankshafts, the actual part shapes sometimes become so complicated in programming by conventional CAD/CAM systems that many forging vendors who use CAD/CAM often need to compensate unsuccessfully defined portion of $3 \mathrm{D}$ geometries by outsourcing them to old-fashioned pattern makers. Therefore, the conventional CAD/CAM process ironically increases the amount of hand operation as well as inflating the investment for computer power.

It would be of great benefit for CAD/CAM users to automate the conventional process that has been relying on skilled operator's experience. To meet this ultimate requirement for idealistic CAD/CAM process, we require some advanced software development which would significantly extend the existing technology.

In this study, examples of an automated process of conventional CAD/CAM system are introduced which applies a rule-based intelligent approach in defining 3D surface geometries for crankshaft forgings.

\section{CHARACTERISTICS OF DIE FORGING CRANK-SHAFT}

Crankshaft forging has the following characteristics:

It is a mill product.

Crankshaft is a built-in part of an automotive engine and is normally produced with mold and die making process. It is merely a "raw material" when shipping out from forging vendors and will be afterwards machined and ground at automotive companies into the final engine part.

\section{It is a structural part.}

The crankshaft, which cannot be seen from the outside, is designed such that it should only satisfy mechanical conditions required within engine; structural strength, vibration and noise, and spatial positioning against other parts (piston, con-rod etc.). While it does not need an artistically designed free form surface, it is usually consolidated with primitive solid geometries complicatedly blended together. Those primitive geometries are cylinders, cones, and other "curve-driven surfaces" that are defined by manipulating analytical curves in 3D space.

In this study, three kinds of those curve-driven surfaces were chosen: extruded surfaces, revolved surfaces and ruled surfaces. There are other types of surface in defining crankshaft that is created during inserting a blending surface between those curve-driven surfaces. This type of blended surface is what is called "freeform surface". 
The actual shape of finished crankshaft which is ready to be assembled within engine mainly has such kind of curve-driven surfaces and dose does not contain many free-formed surfaces. However, as-forged crankshafts have many kinds of draft surfaces and blended surfaces (free-formed surface) this consequently make 3D modeling still more difficult.

\section{CAD SYSTEM NEWLY DEVELOPED}

The following items have been considered in developing the automated CAD system for crankshafts:

\subsection{Modeling of basic shape (portions defined by structural consideration)}

As stated earlier, crankshafts have two types of surfaces; curve-driven surfaces (three kinds of analytically defined surfaces) and free-form surfaces (blend surfaces between those curve-driven surfaces). If the crankshaft geometry were built only by curve-driven surfaces, it would be possible to define each of those surfaces parametrically and to transform its shape variationally with ease. But actual crankshaft geometry will definitely contain lots of free-form surfaces (draft surface and blend surface) and it will never be possible to parametrically or variationally handle those geometries by a conventional process.

In this study, a new concept of "procedure oriented constraint handling" has been applied to improve 3D modeling of crankshaft; for better stability of surface blending, quicker response to engineering change, and standardization of modeling operation.

There are two features in conventional modeling technique done by skilled designers that were referenced to this new concept:

\section{Modeling rules}

A conventional process of generating curve-driven surfaces and inserting blend surfaces between those analytically defined surfaces can be controlled by a "modeling process rule" that dispenses with tricky hierarchical structure in the modeling operation and hence makes it much simpler by enabling the designer to handle both types of surfaces in only one level.

\section{Simultaneous treatment of curve-driven surface and blend surface}

Extension of the modeling rule mentioned above makes it possible to treat the curve-driven surfaces and blend surfaces at the same time. A class concept is introduced to define the minimum unit of generating each surface. In this study, two classes to generate curve-driven surface and blend surface have been applied as the new concept CAD system.

A method to define these three types of curve-driven surfaces (extruded surface, revolved surface, and ruled surface) with this class concept is shown in Figure 3.1. 
A newer class for another type of curve-driven surface will be produced by combining those basic classes with a special I/O facility. It then can be replaced by a procedure of connecting these types of classes such that all of those classes be utilized within a specific common domain of crankshaft.

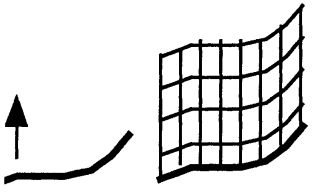

extrude-surf

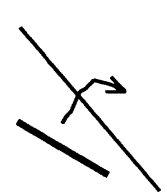

revolved-surf
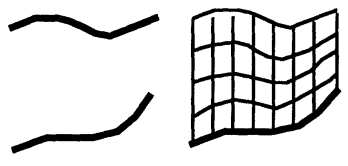

ruled-surf

Figure 3.1 Three kinds of generation classes

Figure 3.2 shows an example of a basic class called JOURNAL that defines the surface on the main journal side of crankshaft counterweight. This class requires two kinds of data input operation; geometry data in IGES format called JOURNAL and the draft angle value.
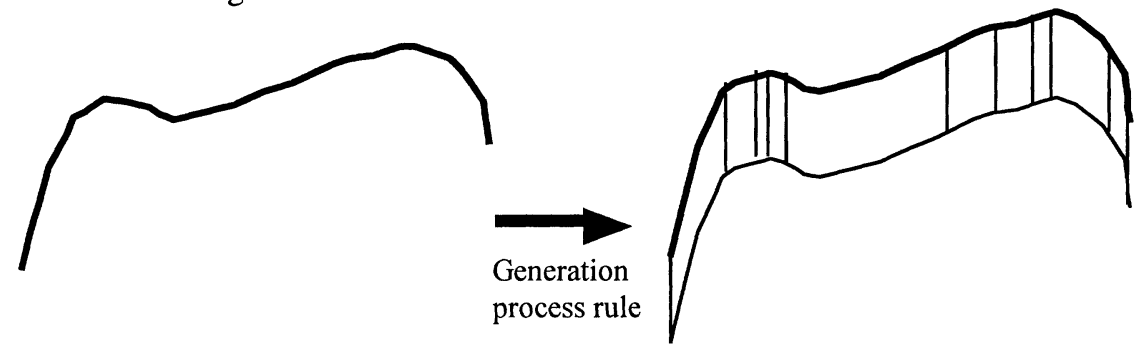

IGES format data (JOURNAL Curve) added value of drawing incline a die forging

Curved surface that has drawing incline of a die forging (JOURNAL Surface)

Figure 3.2 An example of curved surface generation process rule to obtain the drawing incline of a die forging.

In a manner stated above, every class library can be defined for the total domain of crankshaft. Class libraries that are actually used for production will be created by arranging this concept with field information from skilled operators which is collected by interviewing them upon their own modeling rules they have within their know-how. 


\subsection{Class library (repository) of modeling process of various curved surfaces}

A method is explained to make a new curved surface generation class, by combining the basic curved surface generation class with the I/O facility for curved surface generation. Three basic curved surface generation classes, extrude-surf, revolved-surf and ruled-surf are shown in Figure 3.1.

At first, a new curved surface generation class is made by combining basic curved surface generation classes. Next, it can be replaced by the procedure of connecting this class to the curved surface generation process of the die forging crank-shaft modeling domain. An example which uses one of the basic curved surface generation classes, the extrude-surf, is shown in Figure 3.2. Extrude-surf forms a curved surface of a surface called JOURNAL. This basic generation class uses two input values, the geometry shape of IGES format called JOURNAL and the incline value of the die forging.

By using this method it is possible to build the class library. A curved surface generation process rule, can be arranged by the modeling process that craftsmen can specifically state with the given rule format in addition to the above procedure.

\subsection{Blended surface}

The surface blending process to generate a 3D surface of crankshaft using this new concept CAD system is as follows:

1. A $2 \mathrm{D}$ basic contour is drawn in $3 \mathrm{D}$ space.

2. A 3D wire-frame is defined by manipulating this basic contour in 3D space.

3. Other main geometric elements are defined that are commonly used in crankshaft such as draft surface and parting plane. Curve-driven surfaces that would overwhelm the whole model of crankshaft are also defined at the same time in the forms of extruded surface, revolved surface, and ruled surface.

4. The created model in this manner is trimmed and blended such that unnecessary portions of surfaces be eliminated and every sharp corner be rounded.

Among the four steps above, process 4 is the most important in actual modelling of crankshaft.

Although there may be another argument that the lead time of 3D modeling process could be shortened by replacing the conventional type of surface (Bezier etc.) with NURBS (Non-Uniform Rational B-spline), it is not a fundamental solution in improving the modeling process itself. In actual modeling operation, it would not be sometimes easy to blend even simple-shaped edge. Figure 4.1 is an example which shows that blending cannot be completed on a edge where two curves (one built with a series of arcs and the other with line and arc) meet 
together. In this case, smooth fillet cannot be fitted in due to too large radius against the length of the curves.

\section{DIE FORGING MODELING PROCESS}

A typical procedure of generating three dimensional curved surface shapes of a die forging crank-shaft, using the developed CAD system, is as follows:

I. Making a two dimensional fundamental contour in the three dimensional space.

II. Making the three dimensional wire-frame model by developing the above contour figure in the three dimensional space.

III. Modeling typical geometric elements seen especially in die forging products, such as incline sides and parting sides. The analytical curved surfaces that fundamentally cover the whole model are at the same time defined as the wireframe model. These analytical curved surfaces consist of extrude surfaces, revolved surfaces and ruled surfaces.

IV. Trimming and filleting the modelled geometry, which cuts the unnecessary parts, and rounds the sharp edges between the modelled curved surfaces.

From the above, process IV, has the major importance in this modeling procedure of three-dimensional die forging geometries.

It has been demonstrated that the lead time could be shortened by half when shifting from Beizer curved surface to NURBS (Non-Uniform Rational B-spline Surface), in the three dimensional curved surface modeling procedure.

But it is not enough to improve the performance of the surface modeling system. It is required to introduce the knowledge of skilled craftsmen into the CAD system. The modeling process took into account the craftsmen expertise, using it as an important part of the geometric modeling system based on NURBS. This led to an efficient, reliable and fast modeling activity, performed by the developed CAD system. Several examples show the applicability and availability of the developed system.

In real die shape, it may not be possible to insert a fillet even in a simple edge shape. For example, when an edge is formed by a series of an arc, a linear line and an arc, as the one shown in Figure 4.1a, a smooth fillet will not fit in, because the radius is too big for the length of the linear line. A mathematical inconsistency occurs when trying to adjust the fillet's radius to the length of the linear line.

In this situation, craftsmen usually remove the linear line from the edge, using modeling knowledge, and then directly join one arc with another. This modeling knowledge can simplify the die shape and it is practical in use. It is convenient to handle the above-mentioned procedure as the modeling rule shown in Figure 4.1b. 

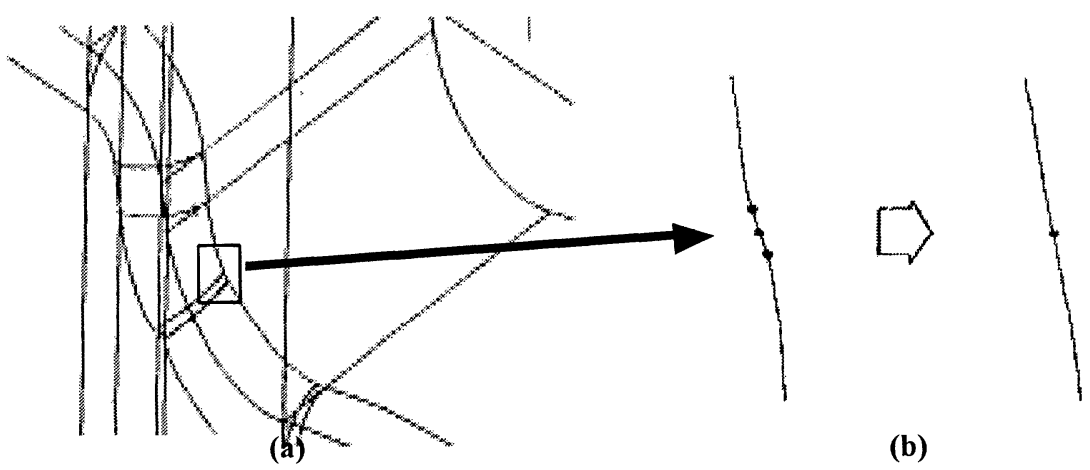

(b)

Figure 4.1 An example to revise the outline shape

\section{NEW-CONCEPT CAD SYSTEM}

The CAD system introduced in this study with a new concept was developed based on the architecture shown in Figure 5.1 which consists of three units; 3D CAD/CAM module, intelligent CAD construction tool, and automatic modeling module.

At first, 2D contour is drawn in 3D space using the 3D CAD/CAM module (3D CAD that is commercially available) as shown in Figure 5.2a. A surface blending operation will then be added with the automatic modeling module that was newly developed for this study (Figure 5.2b). Finally, an optional operation is added again with the $3 \mathrm{D}$ CAD/CAM module for any exceptional case when knowledge capturing was not successfully done beforehand. In this case, the miss-treated portion of the model is repaired by conventional 3D CAD operation.

\subsection{System flow}

Adding to the system procedure described in the previous section, its system flow will be briefly explained below:

- Module "Basic Model" first generates basic elements of curve-driven surfaces and trims them.

- Module "Filleted Model" then adds surface blending based on the modeling rules.

- Module "Interactive Model" gives alternative process of interactively adding blended surface onto the model.

- IGES formatted data and other various parameters related with are stored in the data base. 
- Finally "3D CAD/CAM" gives an option to modify the model interactively same as skilled operators will do on conventional 3D CAD systems.

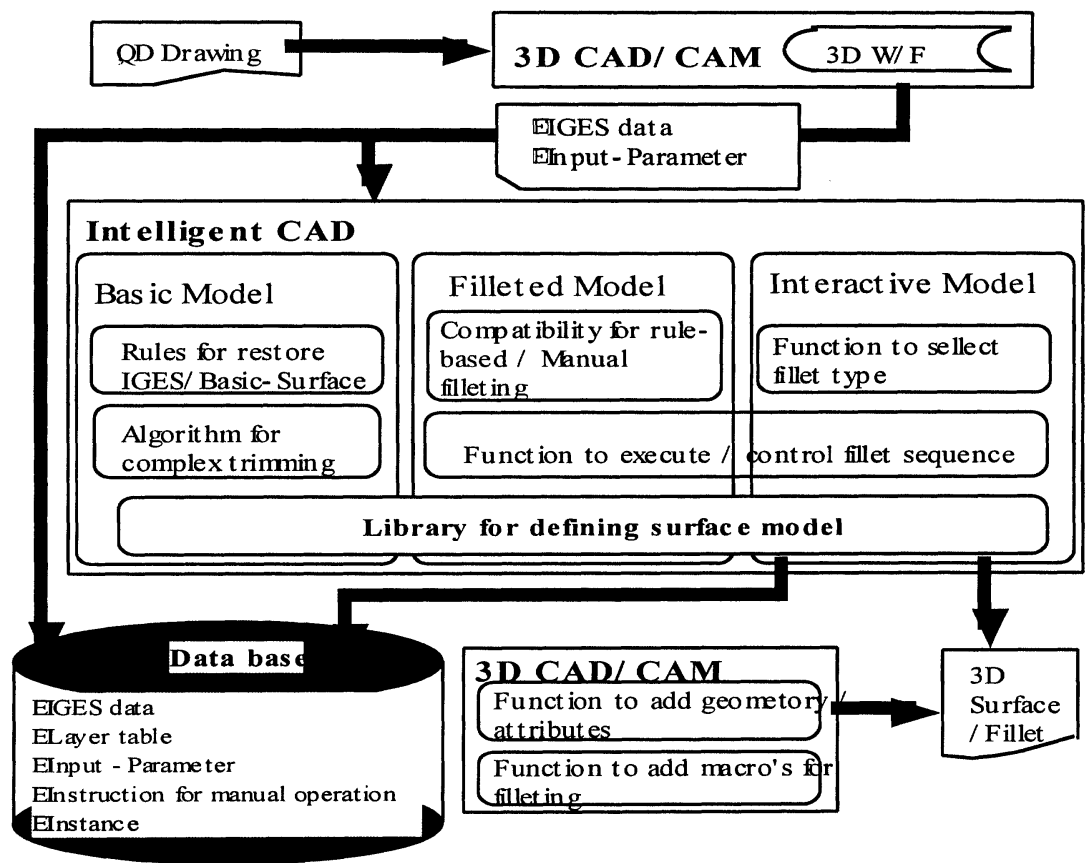

Figure 5.1 Crank-shaft CAD System

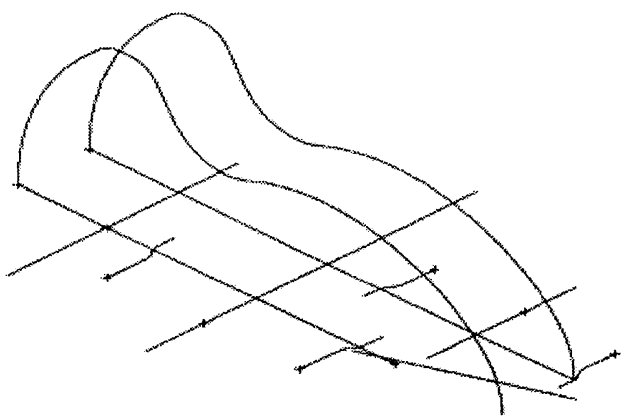

Figure 5.2a Interactive shape generation 


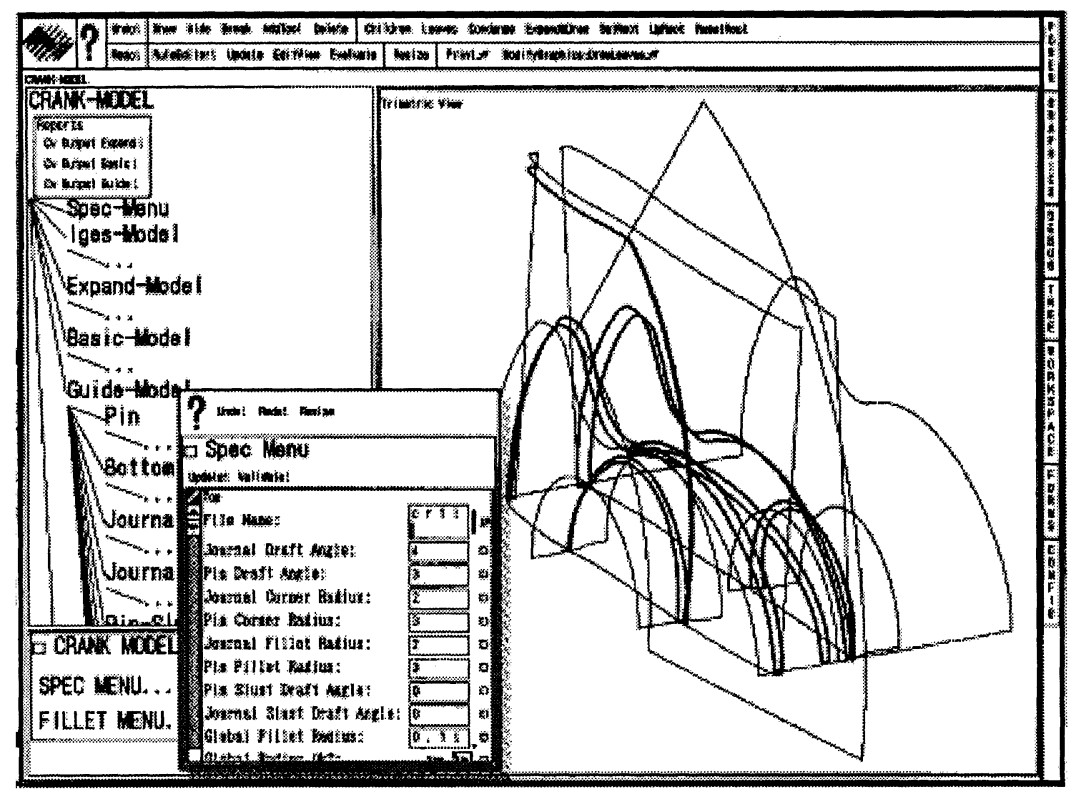

Figure 5.2b Automatic modeling module

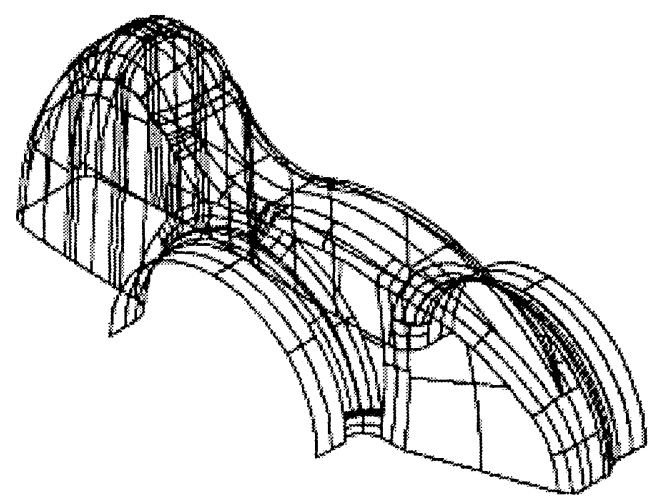

Figure 5.2c Result of die forging model

\subsection{Function of each module}

The developed modules in the CAD system are shown in this section. LIBRARY ("Library for defining the surface model") 
This function is a very important module that determines all of the basic tree structure of the whole model. There are two types of classes that define curvedriven surfaces of crankshaft; a group of functions to generate basic surfaces (surface definition, surface blending, and surface trimming) and extended application for creating other complexly blended surfaces along a series of surface patches that are particularly used for crankshaft.

When a library gets ready, the whole shape of crankshaft can be described in a special program format and any other modeling knowledge can be easily added to the library.

\section{RULE ("Rules to restore IGES/basic surface")}

This function intelligently handles two types of CAD data; geometrical data of 3D CAD in IGES and attribute data in text format.

By activating the classes of curve-driven surfaces, data table of such rules that are already prepared will give a rough geometry of the model (without the blend) and then add the blend.

At first, each geometric element and its corresponding attribute are imported from IGES data and text data such that those combination can be explicitly identified by the layer number. Basic elements of curve-driven surfaces will then be defined by attribute data described by rules and data list that can be altered by skilled operators.

\section{"Algorithm for complex trimming"}

A provisionally blended surface with a small radius is defined where there is an intersection between the surfaces as shown in Figure 5.2d. Unnecessary portions of those surfaces will then be trimmed off to generate the Basic Model (rough model without blend).

\section{"Function to execute/control fillet sequence"}

When there occur some difficulties in trying surface blending in generating a Filleted Model, this function with higher priority to "Rules for restore IGES/basic surface" is executed to automatically rearrange the order of modeling steps within the rules or to change the classes instead of having operators vary the initial values.

\section{"Compatibility for rule-based/manual filleting"}

The modeling rules and the order of modeling steps can also be interactively controlled by this function (with higher priority to "Rules for restore IGES/basic surface") when operators indicate the change of the blending process and the addition of check items. 


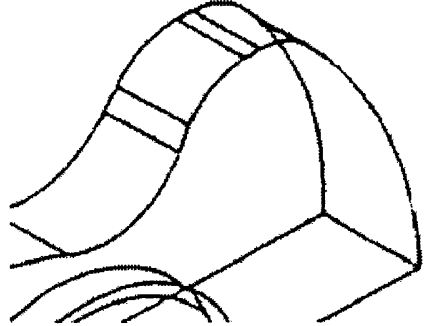

(a) Fillets of minute radius

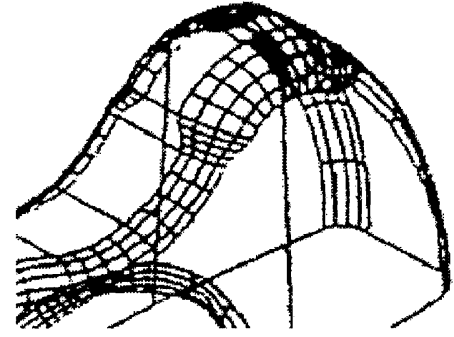

(b) Fillets of normal radius

Figure 5.2d Algorithm for complex trimming

\section{GUI(Graphical User Interface}

"Function to select fillet type"

This function is a module used for blending and trimming simultaneously for two curve-driven surfaces as shown in Figure 5.2b. It is mainly used when making a change in the blend type interactively; from fixed radius fillet to variable radius fillet for instance.

"Function to add macro for filleting (Source Code Generator)"

This function is a module used for generating macro programs by utilizing conventional surface classes interactively for difficult blending operation with the rule base or with an existing blending procedure.

\section{CAD/CAM Modules(consists of the following two functions}

"Function to add geometry/attributes"

This function is a module used for capturing geometric data and attribute data from drawings. It actually works in this study on a 3D CAD system to output attributes as an intermediate file and geometric shape data as an IGES file.

\section{"Data base"}

All information created as stated above is stored/restored in external devices (magnetic disks) in ASCII format with this function module. 
A new-concept CAD system, developed in this study, would automate the conventional process of crankshaft forgings, designed those proved capable of being practically used for actual CAD/CAM operation with good enough productivity.

Several types of curve-driven surfaces were used to test the possibility of automating the 3D modeling process of crankshaft.

However, it is still required to capture more knowledge when this system is to be applied to the actual field operation by veteran operators.

\section{REFERENCES}

F. Kimura et al.: Variational Product Design by Constraints Propagation And Satisfaction in Product Modeling, Ann. CIRP, 35, 1 (1986).

F. Kimura: Product and Process Modeling as a Kernel for Virtual Manufacturing Environment, Annals of CIRP, Vol43, No.1 (1994).

H. Masuda et al.: A Mathematical Theory an Application of Non-Manifold Geometric Modeling, Advanced Geometric Modeling for Engineering Application, North-Holland (1990).

H. Suzuki et al: Treatment of Dimensions on Product Modeling Concept, Design and Synthesis, North-Holland, pp491-496 (1985).

M. Nishimura et al: CAD/CAM of the spot (in Japanese), daily publication industry newspaper publishing company, pp.79-109.(1992)

Mantyla: An Introduction to Solid Modeling, Conference Science Press (1988).

R,E. Phillips and L. W. Rosenfeld : A Knowledge Based for Design Automation, 6th Int. Symp. on Offshore Mechanics and Artic Eng. (1987).

R. Gotoh: Three dimensional CAD/CAM getting started $(1990,1991)$ (in Japanese), Nikkei CG, 1990.12-1991.2.

S. Tanimoto: A mold and die production shape modeller (in Japanese), UNISYS technical report, Vol.37, No. 5, pp87-116 (1993)

T. Suzuki: Problems of solid modeller and development of curved surface solid coexistence modeller (in Japanese), precise engineering bulletin, Vol.56, No. $11, \mathrm{pp} 2015-2020$ (1990)

Y. Murakami et al: Development of railroad chassis CAD system on the basis of product model (in Japanese), precise engineering bulletin, 1992.06, pp967972.

\section{BIOGRAPHY}

Yukimi Murakami, graduated from Tottori University, received a Master of Engineering degree in Mechanical Engineering from Tottori University. He joined Sumitomo Metal Industry and realized research in CAD systems. At present, he is working in the field of CAD system architecture. 
Eiji Arai is graduated from Tokyo University. He is working in the field of CAD system and Factory Automation at Osaka University.

Fumihiko Kimura is graduated from Tokyo University. He is working in the field of CAD system and Factory Automation at Tokyo University. 